\title{
$1.5 \mu \mathrm{m}$ Epitaxially Regrown Photonic Crystal Surface Emitting Laser Diode
}

\author{
Zijun Bian ${ }^{\circledR}$, Katherine J. Rae ${ }^{\circledR}$, Adam F. McKenzie, Ben C. King, Nasser Babazadeh, Guangrui Li ${ }^{\circledR}$, \\ Jonathan R. Orchard, Neil D. Gerrard, Stephen Thoms, Member, IEEE, Donald A. MacLaren, \\ Richard J. E. Taylor ${ }^{\circledR}$, Member, IEEE, David Childs, Member, IEEE, and Richard A. Hogg ${ }^{\circledR}$, Member, IEEE
}

\begin{abstract}
We present an InP-based epitaxially regrown photonic crystal surface emitting laser diode, operating under pulsed electrical drive at room temperature, and lasing at $1523 \mathrm{~nm}$. This opens the route to the development of high efficiency InP based surface emitting lasers.
\end{abstract}

Index Terms-Photonic crystal, semiconductor growth, semiconductor lasers, surface emitting lasers.

\section{INTRODUCTION}

$\mathbf{T}$ HERE has been considerable recent interest in the development of photonic crystal surface emitting lasers (PCSELs) [1]. A photonic crystal (PC) is formed through a $2 \mathrm{D}$ periodic variation in refractive index, and by utilising 2nd order Bragg diffraction a surface emitting laser can be realised [2].

PCSELs have shown single mode operation [3], [4], low divergence [5], polarisation and beam shape control [6], [7], beam steering [8], high power and brightness [9], and coherently coupled arrays [10], [11]. There has also been significant work on the simulation of devices [12]-[15] to optimise output power [16], [17], and more recently simulation results have

Manuscript received September 16, 2020; revised October 21, 2020; accepted November 11, 2020. Date of publication November 18, 2020; date of current version December 4, 2020. This work was supported by the Engineering and Physical Sciences Research Council (EPSRC) Center for Doctoral Training in Photonic Integrations and Advanced Data Storage under Grant EP/L015323/1. The work of Katherine J. Rae was supported by an EPSRC Impact Acceleration Account under Project EP/R511705/1. The work of Adam F. McKenzie was supported by the Royal Commission for the Exhibition of 1851 and CST Global Ltd. The work of Richard J. E. Taylor was supported by the RAEng Enterprise Fellowship. (Corresponding author: Zijun Bian.)

Zijun Bian, Katherine J. Rae, Ben C. King, Nasser Babazadeh, Guangrui Li, Stephen Thoms, Richard J. E. Taylor, David Childs, and Richard A. Hogg are with the James Watt School of Engineering, University of Glasgow, Glasgow G12 8QQ, U.K. (e-mail: z.bian.1@ research.gla.ac.uk; katherine.rae@glasgow.ac.uk; b.king.1@ research.gla.ac.uk; n.babazadeh@ sheffield.ac.uk; guangrui.li@glasgow.ac.uk; stephen.thoms@glasgow.ac.uk; richard.taylor@glasgow.ac.uk; david.childs@glasgow.ac.uk; richard.hogg@ glasgow.ac.uk).

Adam F. McKenzie is with the James Watt School of Engineering, University of Glasgow, Glasgow G12 8QQ, U.K., and also with the CST Global Ltd., Glasgow G72 0BN, U.K. (e-mail: a.mckenzie.1@research.gla.ac.uk).

Jonathan R. Orchard and Neil D. Gerrard are with the CST Global Ltd., Glasgow G72 0BN, U.K. (e-mail: jorchard@ compoundsemi.co.uk; neil.gerrard@glasgow.ac.uk).

Donald A. MacLaren is with the School of Physics and Astronomy, University of Glasgow, Glasgow G12 8QQ, U.K. (e-mail: donald.maclaren@glasgow.ac.uk).

Color versions of one or more figures in this letter are available at https://doi.org/10.1109/LPT.2020.3039059.

Digital Object Identifier 10.1109/LPT.2020.3039059 suggested that speeds of $40 \mathrm{GHz}$ could be achieved from a small, resonator embedded device [18].

Future 5G roll-out requires a corresponding increase in the size of data-centres, all of which require millions of fibre links, both inside them - and between data-centres - serviced by low cost, high reliable, high power lasers operating at $1300 \mathrm{~nm}$ and $1550 \mathrm{~nm}$. PCSEL devices are an ideal candidate for this. Additional applications for such surface emitting InP based devices include free-space communications [19], LiDAR, fibre-based sensing [1], etc.

PCSEL devices have traditionally been realised with wafer fusion [2]-[5], but more recently epitaxial overgrowth through MBE [1], [20] and MOVPE has been utilised [21]-[24]. This move from wafer fusion to epitaxial regrowth has been critical for achieving high output powers [1], [9]. This is because the wafer fusion interface contains regions of discontinuous crystallinity, that result in undesirable defect states [1]. Epitaxial regrowth allows single-crystal PCSEL structures to be realised with encapsulated voids or fully in-filled photonic crystals [21]. Recently, a study of MOVPE growth parameters for optically pumped airhole/InP PCSELs has been reported [25].

In this letter, we demonstrate an epitaxially re-grown InP PCSEL diode operating at $1523 \mathrm{~nm}$ under pulsed electrical injection at room temperature. This builds on work we reported at Photonics West 2020 [26]. The realization of epitaxially regrown PCSELs at this wavelength, as opposed to ones via wafer fusion, is a critical step in providing a route to engineer high-power sources with high beam quality.

\section{Device Design AND Simulation}

Fig.1 shows a schematic of our InP based epitaxially regrown PCSEL structure, whilst table I presents the layer structure. The $\mathrm{PC}$ is a circular atom in a square lattice. Assuming an average refractive index for the PC layer of 3.24, the structure is simulated using the Ritz-Iteration method [27]. The mode shows an overlap with the QWs of $4.7 \%$ and overlap with the photonic crystal of $16.4 \%$.

Fig. 2(a) shows a conventional cross-sectional transmission electron microscope (TEM) image of our regrown photonic crystal grating layer (details later). The bright, central region corresponds to a crystallographic void formed within the etched feature; this is encapsulated laterally by GaInAsP and InP above. Such voids are observed within each of the grating holes. Due to the lattice-matched nature of the structure there 
(a)

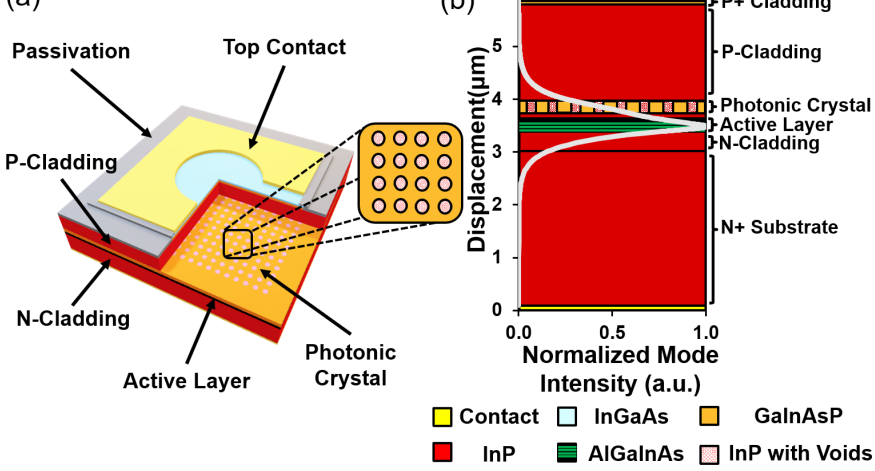

Fig. 1. (a) A schematic of the device, with cut-out area showing the photonic crystal pattern and active layer. (b) A schematic of the corresponding mode distribution inside the device structure. The epitaxial structure is shown, with the various materials in the key below.

TABLE I

$1.5 \mu \mathrm{m}$ PCSEL EPITAXIAL STRUCTURE

\begin{tabular}{|c|c|c|c|c|c|c|}
\hline Layer & Material & $\begin{array}{c}\text { Thickness } \\
\text { (nm) }\end{array}$ & $\begin{array}{l}\text { Strain } \\
(\mathrm{ppm})\end{array}$ & $\begin{array}{c}\text { PL } \\
(\mathbf{n m})\end{array}$ & $\begin{array}{c}\text { Doping } \\
\text { Concentration } \\
\left(\mathrm{cm}^{-3}\right)\end{array}$ & $\begin{array}{c}\text { Growth } \\
\text { Step }\end{array}$ \\
\hline 17 & $\mathbf{I n}_{0.45} \mathbf{G a}_{0.55} \mathrm{As}$ & 75 & & & $2 \times 10^{19}$ & \multirow{4}{*}{ Regrowth } \\
\hline 16 & $\mathbf{G a}_{0.29} \mathbf{I n}_{0.71} \mathbf{A s}_{0.62} \mathbf{P}_{0.38}$ & $.38 \quad 25$ & & $1300 \pm 25$ & $2 \times 10^{18}$ & \\
\hline 15 & InP & $1.8 \mu \mathrm{m}$ & & & $1 \times 10^{18}$ & \\
\hline 14 & InP & 0.2 & \multicolumn{3}{|c|}{$1 \times 10^{18} \pm 50 \%$} & \\
\hline 13 & $\mathbf{G a}_{0.22} \mathbf{I n}_{0.78} \mathbf{A s}_{0.48} \mathbf{P}_{0.52}$ & 243 & -300 & $1200 \pm 25$ & $1 \times 10^{18}$ & PC layer \\
\hline 12 & InP & 50 & & & $8 \times 10^{17}$ & \multirow{12}{*}{$\begin{array}{c}\text { PCSEL } \\
\text { based } \\
\text { structure }\end{array}$} \\
\hline 11 & InP & 50 & & & $6 \times 10^{17}$ & \\
\hline 10 & $\mathrm{Al}_{0.45} \mathbf{G a}_{0.02} \mathbf{I n}_{0.53} \mathrm{As}$ & 90 & -300 & & $4 \times 10^{17}$ & \\
\hline 9 & $\mathbf{A l}_{0.38} \mathbf{G a}_{0.09} \mathbf{I n}_{0.53} \mathrm{As}$ & 15 & -300 & & $3 \times 10^{17}$ & \\
\hline 8 & $\mathbf{A l}_{0.26} \mathbf{G a}_{0.25} \mathrm{In}_{0.49} \mathrm{As}$ & is 12 & -3000 & $1050 \pm 15$ & Undoped & \\
\hline 7 & $\mathbf{A l}_{0.26} \mathrm{Ga}_{0.25} \mathrm{In}_{0.71} \mathrm{As}$ & s $6 \pm 5 \%$ & 12000 & 1530 & Undoped & \\
\hline 6 & $\mathrm{Al}_{0.26} \mathrm{Ga}_{0.25} \mathrm{In}_{0.49} \mathrm{As}$ & is 8 & -3000 & $1050 \pm 15$ & Undoped & \\
\hline 5 & $\mathrm{Al}_{0.26} \mathbf{G a}_{0.25} \mathbf{I n}_{0.49} \mathrm{As}$ & 12 & -3000 & $1050 \pm 15$ & Undoped & \\
\hline 4 & $\mathbf{A l}_{0.40} \mathbf{G a}_{0.08} \mathbf{I n}_{0.53} \mathrm{As}$ & 15 & -300 & & $6 \times 10^{17}$ & \\
\hline 3 & $\mathbf{A l}_{0.43} \mathbf{G a}_{0.05} \mathbf{I n}_{0.53} \mathrm{As}$ & 15 & -300 & & $1 \times 10^{18}$ & \\
\hline 2 & InP & 300 & & & $1 \times 10^{18}$ & \\
\hline 1 & InP & $3 \mu \mathrm{m}$ & & & $1.5 \times 10^{18}$ & \\
\hline
\end{tabular}

(a)

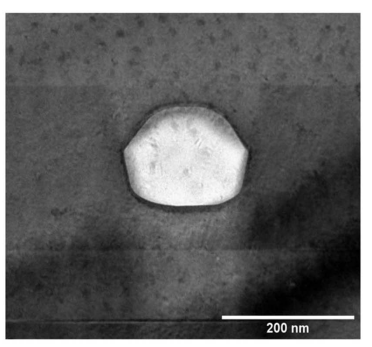

(b)

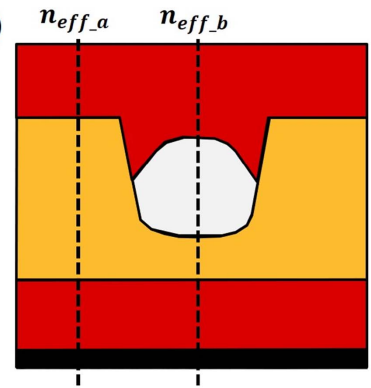

$\square \quad \ln P$

GalnAsP
Voids

AlGalnAs

Fig. 2. (a) A TEM image, and (b) A schematic of one re-grown circular etched feature in the photonic crystal pattern. The etched GaInAsP can be seen, as well as the regrown InP that fills this etched feature, and a central airvoid. The dotted lines in b) denote the cross section from which the effective refractive indices used in further simulations are taken.

is minimal contrast between the GaInAsP layer and adjacent InP layers. Fig. 2(b) shows a schematic of the TEM image shown in Fig. 2(a). The band structure is calculated by 2D plane-wave (PW) expansion method [28] due to the 2D PC

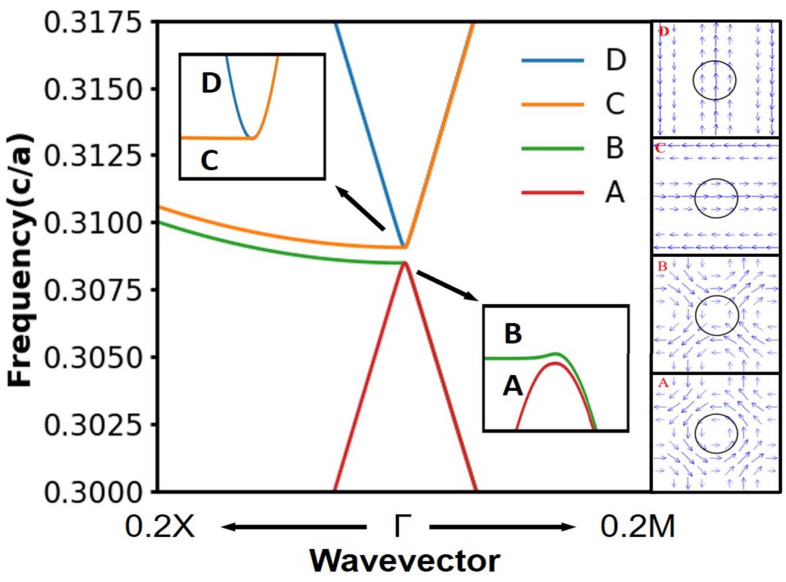

Fig. 3. The band structure around $\Gamma$-point band edges of a square lattice, circular unit cell photonic crystal, with insets showing degeneracy of the bands and the in plane electric field of each band shown adjacent, the black circles represent the edge of the refractive index contrast in the photonic crystal, and the arrow size represents the intensity of the electric field at each point.

structure which requires the mode-index of the PC atom and field. The dotted lines, denoted $n_{\text {eff_a }}$ and $n_{\text {eff_b }}$ show the cross section through the material stack used to calculate the refractive index of the atom, and field materials in the PC, hereafter referred to as region a and $b$, respectively.

Fig. 3 shows the simulated photonic band structure for our PC calculated utilizing the structural information from Fig. 2, where an r/a of 0.17 (where $r / a$ is the ratio between radius and period of the photonic crystal) is determined, as is the position and height of the void. The k-vector is plotted to $0.2(2 \pi / \lambda)$, which was determined to be the collection angle of the optics used to measure the device (described later). As expected from the symmetry of the PC, four modes are obtained. A splitting of the bands at the $\Gamma$-point results in the high density of states from which lasing may occur. The insets in Fig. 3 show band splitting of modes A and B. Shown adjacently are the in-plane electric field highlighting that bands $\mathrm{C}$ and $\mathrm{D}$ are leaky and bands $\mathrm{A}$ and $\mathrm{B}$ are non-leaky and therefor the likely lasing modes.

\section{DeVice FABRicAtion}

The devices were fabricated on InP epitaxial wafers, designed to emit around $1550 \mathrm{~nm}$ as shown in Table I. On these epitaxial layer structures, $200 \mathrm{~nm}$ of $\mathrm{SiO}_{2}$ was deposited by plasma enhanced chemical vapour deposition. A square lattice, circular unit cell photonic crystal, with a period of $470 \mathrm{~nm}$, was defined by electron-beam lithography in PMMA and etched into the $\mathrm{SiO}_{2}$ by reactive ion etching with $\mathrm{CHF}_{3} / \mathrm{Ar}$ chemistry. This acts as a hard mask for the etching of the underlying semiconductor. Through this hard mask, the semiconductor is etched to a depth of approximately $170 \mathrm{~nm}$ - just above the p-cladding layer atop the AlGaInAs active region with a $\mathrm{CHF}_{4} /$ $\mathrm{H}_{2}$ - based inductively coupled plasma etch. This is slightly lower than the optimal PC atom height of $235 \mathrm{~nm}$ [29]. SEM images of test structures indicate that the etched InGaAsP PC layer is not modified by the regrowth process. See schematic in Fig. 2(b).

The $\mathrm{SiO}_{2}$ hard mask is then removed and epitaxial regrowth is undertaken. Immediately prior to regrowth, the wafer is 

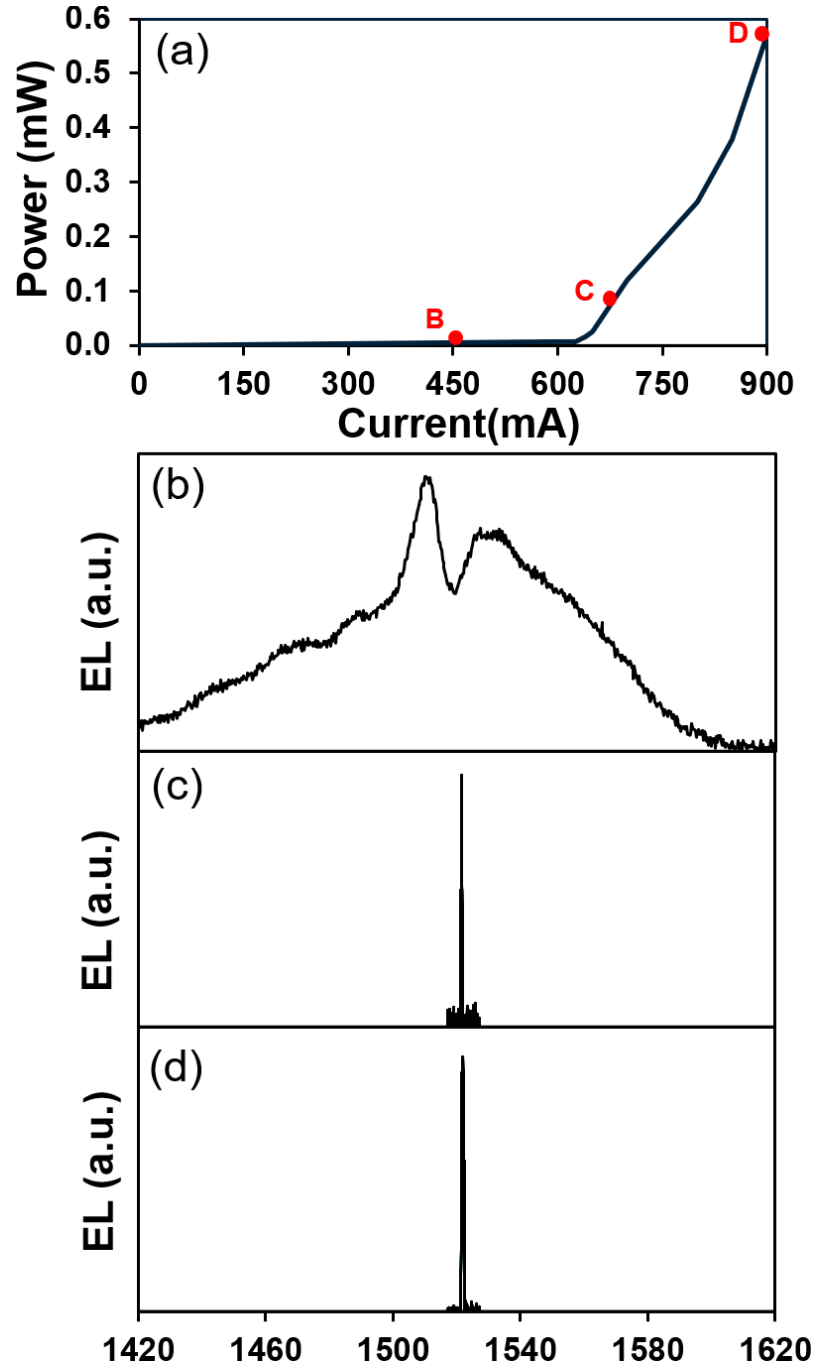

Fig. 4. (a) The current-power characteristics of an InP-based PCSEL, measured under pulsed conditions (10\% duty cycle, $10 \mu$ s pulse width). (b), (c) and (d) show the emission spectra of the device when driven at $450 \mathrm{~mA}, 700 \mathrm{~mA}$, and $900 \mathrm{~mA}$ respectively.

uv/ozone cleaned (UVCOS uv/ozone cleaner) followed by 1 minute in 10:1 buffered HF. Regrowth was performed in an AIXTRON 2400 G2 Planetary MOVPE reactor at 100 mbar, utilising trimethylindium (TMIn) and trimethylgallium (TMGa) as group-III precursors, and phosphine $\left(\mathrm{PH}_{3}\right)$ and arsine $\left(\mathrm{AsH}_{3}\right)$ as group- $\mathrm{V}$ sources. Initially $243 \mathrm{~nm}$ of $\mathrm{InP}$ was deposited at a growth rate of $10 \mathrm{~nm} / \mathrm{min}(32 \mu \mathrm{mol} / \mathrm{min}$ TMIn) at a calibrated reactor temperature of $600{ }^{\circ} \mathrm{C}$. The initial $\mathrm{V} / \mathrm{III}$ ratio of the infilled InP is 814 , it is then reduced to 385 . Following this, $1.8 \mu \mathrm{m}$ of p-doped InP was grown with a V/III ratio of 119 .

Following re-growth, a $200 \mu \mathrm{m} \times 200 \mu \mathrm{m}$ square mesa is patterned with photolithography and etched in a solution of sulphuric acid and hydrogen peroxide. This etches the regrown material, to a depth of $100 \mathrm{~nm}$. A $200 \mathrm{~nm}$-thick $\mathrm{SiO}_{2}$ passivation layer is then deposited across the quarter wafer, with contact windows etched into this with a $\mathrm{CHF}_{3}$ and $\mathrm{Ar}$ containing reactive ion etch. Following the opening of these contact windows, Ti/Pt/Au is deposited on the top surface of the quarter wafer. A $200 \mu \mathrm{m}$ square contact with a "lollipop" shaped aperture of $100 \mu \mathrm{m}$ diameter in the centre

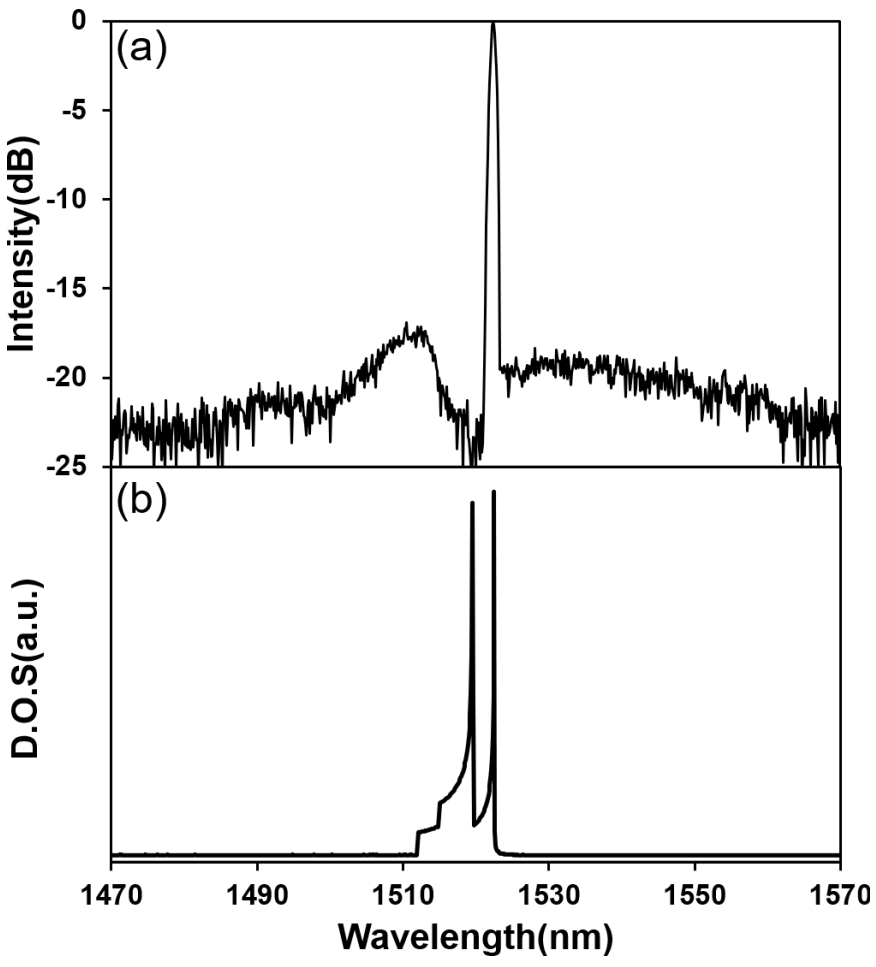

Fig. 5. (a) The emission spectrum of a typical device, plotted from $1470 \mathrm{~nm}$ to $1570 \mathrm{~nm}$. (b) The corresponding simulated optical density of states.

is created via a conventional metal lift-off process, the contact shape is illustrated in Fig. 1(a). $57 \%$ of the device emission area is covered by this contact. A Ni/Au/Ge/Ni/Au n-type contact is then deposited on the bottom side of the quarter wafer, via electron-beam evaporation. This is then annealed at $400{ }^{\circ} \mathrm{C}$ for 1 minute. Thick Ti/Au bond-pads are added to the top surface of the quarter wafer, by an electron-beam evaporation and lift-off process.

\section{RESULTS}

Devices were measured at $15{ }^{\circ} \mathrm{C}$ under pulsed conditions using a $10 \%$ duty cycle and $10 \mu$ s pulse width. Surface emission was collected using a NA $=0.34$ lens, and was focused into a multi-mode fibre-optic cable.

Fig. 4(a) shows the current-power characteristics of a typical device showing a threshold current of $640 \mathrm{~mA}$ $\left(\mathrm{J}=1.6 \mathrm{kA} / \mathrm{cm}^{-2}\right)$. An average slope efficiency of $\sim 0.002 \mathrm{~W} / \mathrm{A}$ is obtained which is low due to the circular symmetry of the PC [2], [16] and the masking of the PCSEL emission by the contacts. Fig. 4(b) shows the sub-threshold electro-luminescence at $450 \mathrm{~mA}$. Two main peaks are observed at $1512 \mathrm{~nm}$ and $1527 \mathrm{~nm}$, attributed to the modification of the spontaneous emission spectrum by the PC. Fig. 4(c) and (d) show the electroluminescence spectra of the same device at $700 \mathrm{~mA}$ and $900 \mathrm{~mA}$, respectively. A narrow peak $(0.34 \mathrm{~nm}$ at $700 \mathrm{~mA}$ with measurement resolution of $0.1 \mathrm{~nm}$ ) is observed at $1522.5 \mathrm{~nm}$ and is attributed to laser emission. A simulated emission wavelength of $1523.2 \mathrm{~nm}$ is obtained, in good agreement with simulation. Fig. 5(a) shows the EL spectrum of the PCSEL with $2 \mathrm{~nm}$ resolution over a narrower spectral range at $900 \mathrm{~mA}$. 
Fig. 5(b) shows the simulated optical density of states (ODOS) for our photonic crystal. The 2 peaks are attributed to the 2 band edges of the photonic crystal. This ODOS is calculated by integrating the simulated band structure over the range of k-vectors that are expected to be collected through our measurement system $(\mathrm{NA}=0.34)$. The observed features are in good agreement with the peak position of the long wavelength peak showing excellent agreement with the experimental spectra shown in Fig.5 (a), which are the non-leaky modes shown in Fig. 3.

\section{SUMmaRY}

We have reported the realisation of an epitaxially regrown PCSEL at $1.5 \mu \mathrm{m}$, operating pulsed at room temperature opening a new route to InP-based surface emitting lasers.

\section{ACKNOWLEDGMENT}

This article was submitted for review, in April 2020. Zijun Bian would like to gratefully acknowledge financial support received from the Engineering and Physical Sciences Research Council (EPSRC).

\section{REFERENCES}

[1] K. Ishizaki, M. De Zoysa, and S. Noda, "Progress in photonic-crystal surface-emitting lasers," Photonics, vol. 6, no. 3, p. 96, Aug. 2019.

[2] Y. Kurosaka, K. Sakai, E. Miyai, and S. Noda, "Controlling vertical optical confinement in two-dimensional surface-emitting photonic-crystal lasers by shape of air holes," Opt. Express, vol. 16, no. 22, p. 18485, 2008.

[3] M. Imada, S. Noda, A. Chutinan, T. Tokuda, M. Murata, and G. Sasaki, "Coherent two-dimensional lasing action in surface-emitting laser with triangular-lattice photonic crystal structure," Appl. Phys. Lett., vol. 75, no. 3, pp. 316-318, Jul. 1999.

[4] K. Sakai, E. Miyai, T. Sakaguchi, D. Ohnishi, T. Okano, and S. Noda, "Lasing band-edge identification for a surface-emitting photonic crystal laser," IEEE J. Sel. Areas Commun., vol. 23, no. 7, pp. 1335-1340, Jul. 2005.

[5] D. Ohnishi, T. Okano, M. Imada, and S. Noda, "Room temperature continuous wave operation of a surface-emitting two-dimensional photonic crystal diode laser," Opt. Express, vol. 12, no. 8, p. 1562, 2004.

[6] R. J. E. Taylor et al., "Mode control in photonic crystal surface emitting lasers through external reflection," IEEE J. Sel. Topics Quantum Electron., vol. 23, no. 6, pp. 1-8, Nov. 2017.

[7] S. Noda, M. Yokoyama, M. Imada, A. Chutinan, and M. Mochizuki, "Polarization mode control of two-dimensional photonic crystal laser by unit cell structure design," Science, vol. 293, no. 5532, pp. 1123-1125, Aug. 2001.

[8] Y. Kurosaka et al., "On-chip beam-steering photonic-crystal lasers," Nature Photon., vol. 4, no. 7, pp. 447-450, Jul. 2010.

[9] K. Hirose, Y. Liang, Y. Kurosaka, A. Watanabe, T. Sugiyama, and S. Noda, "Watt-class high-power, high-beam-quality photonic-crystal lasers," Nature Photon., vol. 8, no. 5, pp. 406-411, May 2014.

[10] R. J. E. Taylor et al., "Coherently coupled photonic-crystal surface-emitting laser array," IEEE J. Sel. Topics Quantum Electron., vol. 21, no. 6, pp. 493-499, Nov. 2015.
[11] R. J. E. Taylor et al., "Electronic control of coherence in a two-dimensional array of photonic crystal surface emitting lasers," Sci. Rep., vol. 5, no. 1, pp. 1-6, Oct. 2015.

[12] R. J. E. Taylor, D. M. Williams, J. R. Orchard, D. T. D. Childs, S. Khamas, and R. A. Hogg, "Band structure and waveguide modelling of epitaxially regrown photonic crystal surface-emitting lasers," J. Phys. D, Appl. Phys., vol. 46, no. 26, 2013, Art. no. 264005

[13] R. J. E. Taylor, P. Ivanov, G. Li, D. T. D. Childs, and R. A. Hogg, "Optimisation of photonic crystal coupling through waveguide design," Opt. Quantum Electron., vol. 49, no. 2, p. 47, Feb. 2017.

[14] G. Li, J. Sarma, R. J. E. Taylor, D. T. D. Childs, and R. A. Hogg, "Modeling and device simulation of photonic crystal surface emitting lasers based on modal index analysis," IEEE J. Sel. Topics Quantum Electron., vol. 25, no. 6, pp. 1-9, Nov. /Dec. 2019.

[15] S. Johnson and J. Joannopoulos, "Block-iterative frequency-domain methods for Maxwell's equations in a planewave basis," Opt. Express, vol. 8, no. 3, p. $173,2001$.

[16] Y. Kurosaka et al., "Band structure observation of 2D photonic crystal with various V-shaped air-hole arrangements," IEICE Electron. Express, vol. 6, no. 13, pp. 966-971, 2009.

[17] K. Sakai, E. Miyai, and S. Noda, "Coupled-wave model for square-lattice two-dimensional photonic crystal with transverse-electric-like mode," Appl. Phys. Lett., vol. 89, no. 2, pp. 7-10, 2006.

[18] T. Inoue, M. Yoshida, M. D. Zoysa, K. Ishizaki, and S. Noda, "Design of photonic-crystal surface-emitting lasers with enhanced in-plane optical feedback for high-speed operation," Opt. Express, vol. 28, no. 4, p. 5050, 2020.

[19] S. Pinna, H. Zhao, S. T. Š. Brunelli, B. Song, F. Sang, and J. Klamkin, "High-power integrated indium phosphide transmitter for free-space optical communications," presented at the Asia Commun. Photon. Conf. (ACPC), Nov. 2019.

[20] M. Nishimoto, K. Ishizaki, K. Maekawa, K. Kitamura, and S. Noda, "Air-hole retained growth by molecular beam epitaxy for fabricating gaas-based photonic-crystal lasers," Appl. Phys. Express, vol. 6, no. 4 pp. $1-5,2013$

[21] D. M. Williams et al., "Epitaxially regrown GaAs-based photonic crystal surface-emitting laser," IEEE Photon. Technol. Lett., vol. 24, no. 11, pp. 966-968, Jun. 1, 2012.

[22] R. J. E. Taylor et al., "Photonic crystal surface emitting lasers based on epitaxial regrowth," in Proc. Conf. Lasers Electro-Opt. Pacific Rim (CLEOPR), 2013, pp. 15-16.

[23] M. Yoshida, M. Kawasaki, M. De Zoysa, K. Ishizaki, R. Hatsuda, and S. Noda, "Fabrication of photonic crystal structures by tertiary-butyl arsine-based metal-organic vapor-phase epitaxy for photonic crystal lasers," Appl. Phys. Express, vol. 9, no. 6, Jun. 2016, Art. no. 062702.

[24] M. De Zoysa et al., "Photonic crystal lasers fabricated by MOVPE based on organic arsenic source," IEEE Photon. Technol. Lett., vol. 29, no. 20 , pp. 1739-1742, Oct. 15, 2017.

[25] C. R. Hedlund, J. M. De Pina, A. Kalapala, Z. Liu, W. Zhou, and M. Hammar, "Buried InP/airhole photonic-crystal surfaceemitting lasers," Phys. Status Solidi Appl. Mater. Sci., Sep. 2020, Art. no. 2000416.

[26] A. F. McKenzie et al., "Advances in regrown all-semiconductor photonic crystal surface-emitting lasers," Proc. SPIE, vol. 11301, Mar. 2020, Art. no. 113010 Y

[27] J. Demmel, Applied Numerical Linear Algebra. Cambridge, U.K.: Cambridge Univ. Press, 1997, pp. 361-366.

[28] M. Plihal and A. A. Maradudin, "Photonic band structure of two-dimensional systems: The triangular lattice," Phys. Rev. B, Condens. Matter, vol. 44, no. 16, pp. 8565-8571, Oct. 1991.

[29] S. Iwahashi, K. Sakai, Y. Kurosaka, and S. Noda, "Air-hole design in a vertical direction for high-power two-dimensional photonic-crystal surface-emitting lasers," J. Opt. Soc. Amer. B, Opt. Phys., vol. 27, no. 6 , pp. 1204-1207, 2010 